\title{
Report of the Research and Survey Committee for Light Source Technology Adapting Semiconductor Ballast
}

\author{
Senichiro Nakanishi \\ (Okayama University)
}

\section{Introduction}

A utilization of electronic device for the operating circuit of discharge lamps not only provided the ballast superior characteristics such as small-size, light-weight and high efficiency but also allowed a development to a variety of operating systems of discharge lamps such as frequency control and waveform control. Furthermore, it is indispensable as an operating circuit for the compact discharge lamp, the alternative to the incandescent lamp, which has been rapidly popularized recently.

Therefore, for a totally balanced higher technological development of a light source system composed of an electronic operating circuit and a discharge lamp and for a promotion of its practical use, it is required, by all means, to research and survey the effect which the operating system of an electronic operating circuit gives to the operating characteristics of discharge lamps.

In addition, not only by adapting the electronic operating circuit to the discharge lamp but also by adapting the discharge lamp to the electronic operating circuit conversely, it is, supposedly, capable of obtaining an operating system provided with small-size, light-weight and high efficiency and function.

From the above-mentioned viewpoint, this committee was installed in May 1987 and an activity of research, survey and discussion of light source technology adapting semiconductor ballast was continued up to May 1989 by holding a committee meeting, a secretary meeting and a study meeting a number of times. Meanwhile, the present situation of electronic operating circuits and discharge lamps was understood and the problems associated thereto were, also, disclosed and, finally, by summarizing the study result on the development of the discharge lamp adapted to the electronic operating circuit, the Committee Report "The Trend and Task of Light Sources Adapting to Electronic Operating Circuits, JIER-015 (1989)" was issued. The committee activity and the summary of the Report will be described in the following.

\section{Members of the Committee (20 members)}

Chairman : S. Nakanishi (Okayama Univ.)

Secretary : M. Ozawa (Matsushita Elec. Ind. Co., Ltd.)

Y. Hirao (Toshiba Lighting \& Techn. Corp.)

Member : R. Itatani (Kyoto Univ.)

K. Ito (NEC Home Electronics, Ltd.)

J. Inagaki (Koito Industries Ltd.)

K. Inui (Toshiba Lighting \& Techn. Corp.)

T. Urayama (Ritsumeikan Univ.)

I. Okuno (Matsushita Electronics Corp.)

K. Kazunaga (Ushio Inc.)

Y. Kemmotsu (Eye Lighting Systems)

M. Saito (Iwasaki Elec. Co., Ltd.)

H. Takasu (Japan Storage Battery)

M. Takahashi (Tottori Univ.)

I. Torii (replaced by S. Ogawa later) (Hitachi, Ltd.)

T. Maeda (Matsushita Elec. Works)

K. Mizuno (Nagoya Inst. of Techn.)

H. Yamazaki (Mitsubishi Elec. Corp.)

M. Ryoko (Akashi College of 
Techn.)

Y. Watanabe (Hitachi Lighting

Co., Ltd.)

\section{Activity of the Committee}

The committee activity was started on Sept. 8,1987 by the first committee meeting (explanation of the object of the committee and discussion of the schedule and the study subjects) and concluded on May 11, 1989 by the 11th committee meeting (final check and approval of the Committee Report). Therebetween, the committee meeting was held every two months in average and the secretary meeting was held four times.

On July 15, 1988 at Okayama University, an open study meeting titled "Lamp characteristics and operating system" was held by the sponsorship of the special study group of light generation and relating system ( 6 reports and about 50 participants).

\section{Outline of the Report}

The outline of the report is described in the following.

Chapter 1 Introduction

Chapter 2 Characteristics and Trend of Discharge Lamp

Mainly, the characteristics and the trend of fluorescent lamps and HID lamps are described.

\subsection{Recent Trend of Lamps}

In the field of HID lamps, an inventive modification of the geometrical structure is reported as a countermeasure against an acoustic resonance at a high frequency operation. In addition, a trend of low power consumption and compactness is noticable.

In the field of fluorescent lamps, lamps adapted for high frequency operation were produced already early in Europe and are now available on the market also in US, but are not sold in Japan.

Additionally, there is a so-called compact lamp which was supplied at first in Europe. In Japan, also, similar lamps are supplied by some manufacturers, but they are not interchangeable with the European products due to the difference of the code and the structure.
Furthermore, it is noticable in fluorescent lamps that amalgam is used as a $\mathrm{Hg}$ source and the diameter of the tube becomes thinner.

\subsection{Characteristics of Discharge Lamps}

(1) Cataphoresis

It means such a phenomenon that an unevenness is found in the luminous color and the luminance when a lamp is operated by DC. In order to prevent this phenomenon, there was invented a variety of countermeasures such as a modification of lamp shape so as to make the tube diameter larger and the tube length longer, a vertical operation and an installation of metal vapor source at the anode side.

(2) Acoustic resonance phenomenon

It is this phenomenon that produces the greatest trouble when electronically operating an HID lamp. It is reported that this phenomenon can be almost avoided by a countermeasure taken at the lamp side such that a modification is given to the tube form so as to provide a concave form in a portion of the lower end of the luminous tube and a frequency modulating operation is used together.

(3) Frequency characteristics of luminous color

The high frequency operation of the fluorescent lamp generates sometimes a luminous color slightly different from the one at the commercial frequency operation. On the other hand, in the HID lamp, the luminous color does not show any change depending on the operating frequency.

(4) Dimming

A possibility of easy realization of dimming function is one advantage attainable in the case of operating the fluorescent lamp by an electronic operating circuit. At the electronic operation it became capable of carrying out a stepwise dimming or a continuous dimming in a relatively simplified way.

(5) Life of lamps

In the case of electronic operating circuit, it is estimated that the life will be extended generally, for example, because a cold cathode starting is avoided by a soft start or because a restriking voltage is low.

(6) Noise

At the high frequency operating of a lamp with a thin tube diameter, a noise is sometimes 
generated by a spike appearing in the waveform of lamp voltage and current. As a countermeasure for preventing this noise, an improvement of the electron emitting performance of the cathode is considerable.

(7) Starting characteristics in electronic operation of fluorescent lamps

In the rapid-start type fluorescent lamp provided with a starting aid by which the maximum value and the frequency of the voltage impressed to the lamp is affected, the higher the frequency, the lower the discharge starting voltage. And the transfer voltage from the glow discharge to the arc discharge depends on the starting aid type. This is to say, in the proximity conductor type, the higher the frequency, the lower the transfer voltage, but in the internal conductive film type, the higher the transfer voltage, conversely.

(8) Restarting characteristics of HID lamps

The discharge restarting voltage of the lamp after the lamp was put out starts to increase just after the putting out, increases gradually for several tenth seconds or one minute and then decreases. In order to be transfered to the main discharge by the power source after the start of discharge, a some degree of current flow is required.

Chapter 3 Characteristics and Trend of Electronic Operating Circuit

The electronic operating circuit is classified, according to the lamp operating frequency or waveform, into DC operation, low frequency square wave operation, high frequency operation, pulse operation, microwave electrodeless discharge and so on. Among them, it is high frequency operating circuit that is supplying a number of products at present.

\subsection{Characteristics of Electronic Operating} circuit

(1) High frequency operating circuit

When the fluorescent lamp is operated by the high frequency operating circuit, not only the lamp luminous efficiency is improved but also the circuit efficiency can be improved, resulting in that a power conservation can be attained. The type of operating circuit is selected by con- sidering the cost or the efficiency. At present, a number of basic circuits as shown below are practically being used.

(A) Single transistor voltage resonance selfexcited inverter: The circuit constitution is simple.

(B) Single transistor voltage resonance separately-excited inverter: It is characterized in the same way as (A).

(C) Two transistor voltage resonance selfexcited inverter: It is also called a constand current push-pull inverter and.has a higher circuit efficiency.

(D) Two transistor current resonance selfexcited inverter: It is also called a seriesinverter. Only a source voltage may be impressed to the switching element but the peak current is large.

(E) Two transistor current resonance separately-excited inverter: It is characterized in the same way as (D).

Furthermore, several inventions were made recently as shown in the following. One example is a high power factor partial smoothing circuit constructed by using an input choke of the constant current push-pull inverter. By converting the lamp current waveform into a pseudo rectangular waveform by using 2 sets of resonance circuit for HID lamps, a generation of acoustic resonance phenomenon was avoided. Concerning the dimming function, an example in which an output is controlled by controlling the conductive time of the switching element of the inverter has been reported. Furthermore, concerning two transistor current resonance self-excited inverter, a variety of improvements of circuit, for example, a progress to an operation of a plurality of lamps and an improvement of driving circuit.

(2) Low frequency square wave operating circuit

This means a type in which the amplitude of lamp current is limited by the low frequency switching circuit. This type has following features: light output is flat; the arc is not going out because a restriking voltage does not appear; and in an HID lamp, an acoustic resonance phenomenon is not produced.

(3) DC operating circuit 
The basic circuit is classified into rising voltage type, dropping voltage type and rising and adopping voltage type. The DC operating circuit is not put in practical use yet because due to cataphoresis the luminous flux is decreased at the positive electrode side in the fluorescent lamp and a color separation is produced in the HID lamp. However, several methods of preventing cataphoresis have been proposed.

(4) Pulse operating circuit

In the high pressure sodium lamp, an impression of pulse will increase the arc current instantaneously, thereby being capable of raising the color temperature of the lamp. Also, an impression of triangular pulse into the lamp with a mixture of $\mathrm{Ne}$ and $\mathrm{Hg}$ will change the rising time of the color temperature, thereby being capable of controlling the luminous color.

(5) Microwave electrodeless discharge

When an electrodeless lamp is placed across the microwave, the sealed-in gas will be excited and emit light. At present, a lamp of high watt is commercialized. The electrodeless discharge operating circuit has several features as follows.

- Quick rising time

- Short lamp reoperating time

- Long life of lamp

- Free selectability of sealed-in gas of lamp

- Small heat radiation from lamp

\subsection{Future Trend}

Hereafter, the following items should be studied.

(1) Higher harmonic wave current of commercial power source

(2) High frequency noise

(3) Infrared noise

(4) Common use of $100 \mathrm{~V}$ and $200 \mathrm{~V}$

(5) Instantaneous restarting of HID lamp

Chapter 4 Requirement Proposed from Light Source to Operating Circuit

\subsection{Fluorescent Lamp}

The fluorescent lamp recently has a trend of small-sizing of lamp as represented by compact self-ballasted fluorescent lamp and compact lamp and the requirement proposed to the operating system such as small-size, light-weight, high efficiency and high luminous flux will be realized by electronic ballast in a higher possibility.

There are many reports concerning the efficacy improvement attained by a high frequency operation. However, some papers report that the luminous output characteristics depend on tube diameter and frequency to a great extent, for example. Therefore, a large allowance can be expected for the optimum design of lamp and its operating circuit.

The higher luminous flux, which can be achieved by increasing lamp current, will improve the efficiency but the electrode will be damaged contrarily. In that case, the damage of the electrode can be decreased, for example, by forming double spots in the electrode.

The problems resulted from the electronic operation of fluorescent lamp and the requirements to the electronic ballast are listed in the following.

(1) Starting

The damage of electrode caused at the starting affects the life of lamp, especially the cold start will shorten the life so that it is required to provide a sufficient preheat current.

(2) Dimming

In fluorescent lamp, too high or too low temperature at the electrode, while being lightened, will introduce a short life so that it is required to keep the electrode temperature properly during dimming.

(3) Lamp current control characteristic

It is required to keep the output characteristics of a fluorescent lamp, which has a large temperature dependancy, at constant by giving the electronic ballast a constant current characteristic.

(4) Standard circuit

A standard circuit provided with a predetermined value of preheat current, impressed voltage at starting, operating frequency and lamp current is required.

(5) High-temperature resistance

A generated heat affects the luminous efficacy and the life of lamp and circuit. Therefore, in the compact self-ballasted fluorescent lamp with a built-in operating circuit, for example, a heat insulation is provided by forming an air layer for heat insulation between the circuit and the lamp. It is required to lower a 
heat generation by increasing the efficiency of the operating circuit and to improve the hightemperature resistance.

(6) Unstable phenomenon at cold temperature

It is well-known that, at the high frequency operation, moving striation is generated in the lamp with sealed-in kripton. It is reported recently that, in compact self-ballasted fluorescent lamp with only sealed-in argon, moving striation was generated. When electronic operating circuits will be more popularized in future, such a phenomenon will probably be generated more due to a variety of combinations of lamps and circuit.

\subsection{HID lamp}

The development of HID lamps is now directed to low power consumption and smallsizing and a variety of requriements is proposed such as higher luminous output, higher efficacy, longer life as well as a correspondence to different color temperatures and higher color rendering properties and no color change during service life. It is expected that the electronic ballast can correspond to these requirements.

In the following, the problems relating to the operation of HID lamps and the requirements or expected items are listed.

(1) Compact and light-weight

It is required that the lamp is compact and light-weight including the discharge stabilizing circuit and the starting circuit. It is reported that the electronic ballast for a recent compact HID lamp has resulted in being decreased to about $50 \%$ in volume and about $30 \%$ in weight compared with the magnetic circuit type ballast.

(2) Efficacy

It is required to improve the system efficacy by raising the efficacy of operating circuit. It is reported that the system efficacy is increased by $15 \%$ by an increase of luminous efficacy by $10 \%$.

(3) Starting and high temperature restarting

In the present compact HID lamp a high voltage is required, especially, for starting and restarting.

(4) Avoidance of unstability caused by acoustic resonance phenomenon
In order to avoid the acoustic resonance phenomenon, a modification of the shape of the lamp was tried but resulted in not being put in practical use. Generally, its countermeasure is taken from the operating circuit side. Recently, a method to control the circuit by detecting the discharge condition is being studied.

(5) Rapid rising of luminous flux

The rising of luminous flux after starting the HID lamp is too slow so that it is required to take a countermeasure thereof. In order to obtain a rapid rising, one example was tried so as to heat the luminous tube by a built-in ceramic heater.

(6) Constant power characteristics, constant color temperature characteristics

It is required depending on the conditions to provide a constant power characteristics, therefore a small color change characteristic, irrespective of source voltage fluctuation, tube voltage change and various conditions encountered during total service life.

Chapter 5 Requirements Proposed from Operating Circuit to Light Source

There are some examples in which a variety of problems relating to the compact and lightweighted electronic operating circuit can be solved by an improvement effectively applicable at the lamp side. Although it might be difficult to apply an improvement to the lamp side, it is required to construct an economical lighting system in cooperation with ballast, luminaire and lamp.

\subsection{Requirement to Fluorescent Lamp}

(1) Starting characteristics

The starting voltage shall be near to the lamp voltage during operation as possible and the starting voltage shall hardly change depending on the ambient temperature change. These characteristics are important for the starting circuit and the protective circuit for the final period of service life.

(2) Phenomenon at the final period of service life

If the discharge is continued even after the service life is reached, not only it gives a stress to the operating circuit but also it sometimes might 
give a damage to the lamp bulb. Although this damage will be usually prevented by a protective countermeasure provided at the circuit side, it is essentially desirable to stop discharge when the lamp could not maintain a normal discharge any more.

(3) Discharge characteristic

If the lamp voltage-current characteristic is linear, a going out of lamp during the dimming can be prevented, thereby simply capable of deepening the degree of dimming.

(4) Temperature characteristic

It is required that a change in the lamp characteristics (luminous flux, moving striation, voltage etc.) shall be small as possible.

(5) Electrode preheating power

It is required that the electrode preheating power at operation and dimming shall be small as possible.

(6) Luminous efficacy

The higher the efficacy, the lower the heat generation, thereby the operating circuit being made compact, resulting in a small-sized luminaire.

\section{(7) Lamp current}

A lower lamp current design makes the current of operating circuit smaller for the lamp with the same power, resulting in an improvement of the efficiency of the operating circuit.

\subsection{Requirements Proposed to HID lamp}

The HID lamp, which has been popularly used mainly for outdoor lighting, has recently extended its application fields, by utilizing its merits of high luminance, high luminous flux, high efficacy and long life, as down-light or spotlight in the space of store, hotel lobby, community area, bank and so on. Together with this trend it is required to make the operating device more compact and light-weighted so that it is directed towards the use of electronic ballast. It is made possible by using an electronic ballast to control dimming, to keep the power constant and to attain a constant color temperature.

In the following the problems relating to the operating circuit and the requirements proposed to HID lamps are listed.
(1) Lamp hardly affected by acoustic

resonance phenomenon

Since the illuminated surface and the light source are comparatively near to each other due to lowpowered lamp, flickering is noticable. It is, therefore, required to take a countermeasure against flickering so that a high frequency operation is expectable. However, on the other hand, an acoustic resonance phenomenon is liable to be generated. In order to prevent it, an operation by using a rectangular wave is an idea but it results in a higher cost. Also, a modification of lamp shape was tried but impracticable yet.

(2) Lamp hardly affected by cataphoresis

When DC operation is used in order to avoid the acoustic resonance phenomenum, a cataphoresis is generated separately as a problem.

(3) Lamp with low starting voltage

Essentially, the starting voltage of HID lamp is high. Furthermore, as it is difficult to install an auxiliary electrode, a very high voltage is required for starting. Therefore, a higher dielectric strength is required for the parts and printed board of the operating circuit so that it is difficult to make the operating circuit smallsized, resulting in that it must be expensive.

(4) Marketing of rated value according to high frequency reference ballast

The official lamp rating is generally determined according to the magnetic circuit type reference ballast. In this case, the lamp voltage is strainfree and has a power factor of $100 \%$ and so that the lamp current has a difference of about $10 \%$.

(5) Lamp whose luminous efficacy and color temperature are hardly affected by dimming.

Chapter 6 Development of Lamp adapted to Electronic Operating Circuit.

\subsection{Low Pressure Discharge Lamp}

In order to discuss a development of low pressure discharge lamp (mainly fluorescent lamp) adapted to the electronic operating circuit, a relationship between the characteristics and design factors of low pressure discharge lamp is shown in Table 1 . In the table, the marking $O$ indicates a relating item and the marking (O) indicates an item to be considered in 
the high frequency operation. Some of the characteristics will be described in the following.

\subsubsection{Starting Characteristic}

The starting process of a fluorescent lamp is the process from the start of discharge to arc discharge, in which the discharge starting voltage is strongly affected by the condition of tube wall. Since the tube wall surface condition can be approximated by a distributed constant circuit and the electric field near the electrode will be increased by an impression of high frequency voltage, the discharge starting voltage will be decreased depending on the increase of frequency. A development of the starting aid system having a low starting voltage by means of an analysis of the electric field within the discharge tube is expectable.

\subsubsection{Operating Characteristic}

(1) Electrode Characteristic

At low discharge frequency, the electrode forms alternatively a negative and a positive space-charge layer at each cycle of anode and cathode, respectively. When the frequency is increased, the ion charge layer formed at the cathode cycle acts as an ion supplying source during the successive short anode cycle and the cycle returns again to the cathode cycle before reaching to an ion shortage. Consequently, since the negative charge layer is not formed and an anode voltage drop is not produced, the anode loss disappears. Thereby, the lamp efficiency will be improved.

As described in the above, it is estimated that the electrode operating condition is remarkably different at commercial frequency and at high frequency, respectively. An analysis of this condition and a development of the electrode adapted to the high frequency operation is desired.

(2) Positive column electron temperature

When the operating frequency becomes higher, the diffusion loss cannot follow up, at first, the change of discharge current and, then, the ionization, also, cannot follow up it. Therefore, at high frequency, the electron density cannot follow up the change of discharge current so that the electron density approaches to a constant value. Since at high frequency the electron density does not change, the change of current introduces a change of electric field. Therefore, the voltage-current characteristic will show a positive resistance characteristic.

\subsubsection{Lamp Adapted to Electronic Operating} Circuit

(1) Lamp adapted to high frequency

The advantage of high frequency is an improvement of luminous efficacy and a stabilization of discharge due to disappearance of restriking phenomenon. The increase of the efficacy is more effective in the discharge tube in which a ratio of the anode drop voltage to the discharge voltage is larger. Also, the discharge tube in which the restriking voltage increases is the discharge tube with a short diffusion length and a thin tube diameter. Therefore, it can be said that the lamp adaptive to the high frequency operation is the lamp in which the diameter is somewhat thin and the discharge length is not so long. As a lamp of this type the compact type fluorescent lamp extensively used recently can be exemplified.

(2) Electromagnetic wave interference

The electromagnetic wave noise generated at the high frequency operation is cassified into the one generated from the operating circuit and the other generated from the discharge tube. The former can be prevented by using a line filter or an electromagnetic shield case. On the other hand, there is not necessarily found an effective countermeasure for the latter. A potential countermeasure is a modification of the shape of the discharge current path and a modification of the constitution of the tube wall material of the discharge tube.

(3) Restriking phenomenon

At the high frequency operation the electron density cannot follow up the current so that the electron density is almost constant during one cycle of the current change. Therefore, the restriking phenomenum disappears. However, even at the high frequency operation, if the degree of smoothness of the above-mentioned partial smoothing circuit is low, the problem of restriking will be produced.

\subsection{HID lamp}

If the HID lamp is operated at high frequency by the electronic operating circuit, a special 
Table 1 Relation Between Design Factors and Characteristics of Low Pressure Discharge Lamp for Lighting

\begin{tabular}{|c|c|c|c|c|c|c|c|c|c|c|}
\hline Characteristics & $\begin{array}{c}\text { Tube dia. } \\
\text { \& dis- } \\
\text { charge } \\
\text { length }\end{array}$ & $\begin{array}{c}\text { Bulb } \\
\text { structure }\end{array}$ & $\begin{array}{c}\text { Starting } \\
\text { aid }\end{array}$ & $\begin{array}{l}\text { Elec- } \\
\text { trode } \\
\text { shape, } \\
\text { emitter }\end{array}$ & $\begin{array}{c}\text { Fluo- } \\
\text { rescent } \\
\text { material }\end{array}$ & $\begin{array}{l}\text { Gas type } \\
\text { and } \\
\text { pressure }\end{array}$ & $\begin{array}{c}\text { Sealing } \\
\text { metal }\end{array}$ & $\begin{array}{l}\text { Coldest } \\
\text { part } \\
\text { temper- } \\
\text { ature }\end{array}$ & $\begin{array}{l}\text { Dis- } \\
\text { charge } \\
\text { current }\end{array}$ & $\begin{array}{l}\text { Elec- } \\
\text { trode } \\
\text { heating } \\
\text { current }\end{array}$ \\
\hline Starting characteristics & 0 & & (Q) & 0 & & 0 & 0 & 0 & & 0 \\
\hline $\begin{array}{l}\text { Life (electrode, luminous flux } \\
\text { holding) }\end{array}$ & O & & O & 0 & 0 & 0 & 0 & 0 & 0 & (0) \\
\hline Restriking voltage & 0 & & & $\mathrm{O}$ & & (O) & 0 & 0 & 0 & $\mathrm{O}$ \\
\hline $\begin{array}{l}\text { Discharge voltage (positive } \\
\text { column electrode drop) }\end{array}$ & 0 & & & (0) & & $\mathrm{O}$ & 0 & $\mathrm{O}$ & () & (O) \\
\hline Efficacy & (0) & & 0 & (0) & 0 & 0 & 0 & 0 & 0 & 0 \\
\hline Luminous color & & & & & (0) & & 0 & 0 & 0 & \\
\hline Discharge stability & (0) & 0 & & & & 0 & 0 & 0 & (C) & 0 \\
\hline $\begin{array}{l}\text { Electromagnetic wave emis- } \\
\text { sion }\end{array}$ & (O) & 0 & & (O) & & 0 & & 0 & (O) & 0 \\
\hline
\end{tabular}

Generally relating item

(9) Item to be considered in the electronic operating circuit

phenomenon different from the one at the operation by the commercial frequency appears depending on the circuit type. Table 2 shows a relationship between the characteristics and design factors of HID lamps. In the table, the marking $O$ indicates a relating item and the marking (9) indicates a item to be considered in the high frequency operation.

\subsubsection{Acoustic Resonance Phenomenon} Generated by High Frequency Operation

When the HID lamp is operated at high frequency, an acoustic resonance phenomenon appears. This appears as an unstable phenomenon of arc, which is a stationary wave generated when the pressure fluctuation in the luminous tube caused by the periodic electrical energy input approaches to the acoustic resonance frequency of the luminous tube. Since the acoustic resonance phenomenon has been in some degree understood quantitatively and qualitatively, it is supposed that an optimum design of the luminous tube which can avoid a resonance phenomenon is expectable. Furthermore, it seems that it is required from the circuit side to improve the stability by optimizing the modulation of the high frequency.

\subsubsection{Drop of Restriking Voltage by High} Frequency Operation

When the HID lamp is operated at the commercial frequency, a restriking voltage is generated at each half-cycle. However, when operated at the high frequency, the duration of zero current becomes short so that it can be said that the high frequency operation as shown above is an operating system advantageous for the HID lamp of low wattage but it is required to design each lamp so as not to lower the luminous tube temperature.

\subsubsection{Cataphoresis Phenomenon at DC}

\section{Operation}

When the HID lamp is operated by DC, especially in the HID lamp with a sealed-in $\mathrm{Ne}$, a color separation is generated by a displacement of $\mathrm{Na}$ ion into cathode. The longer the luminous tube length, the more prominent this trend. A definite relation between $\mathrm{Na}$ vapor pressure and arc length, in which a color separation will not occur, has been obtained and it was confirmed experimentarily.

6.2.4 Color Temperature Rising by Pulse Operation 
Table 2 Relation Between Design Factors and Characteristics of HID Lamp for Lighting

\begin{tabular}{|c|c|c|c|c|c|c|c|c|c|c|c|c|c|c|}
\hline 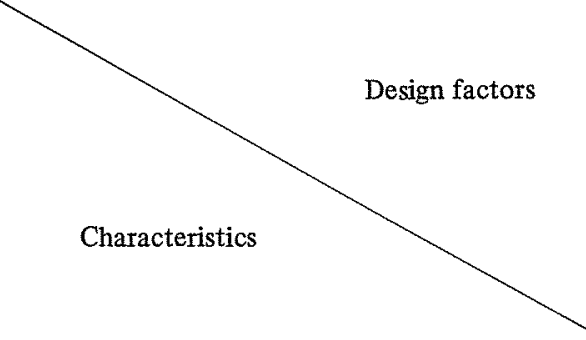 & 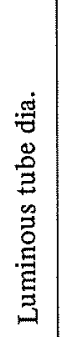 & 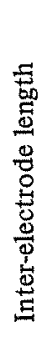 & 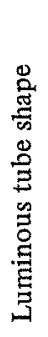 & 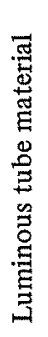 & 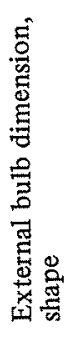 & 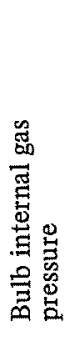 & 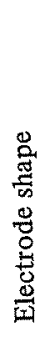 & 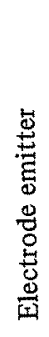 & 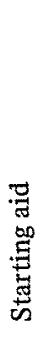 & 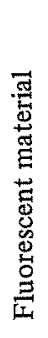 & 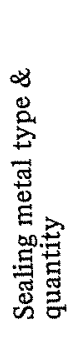 & 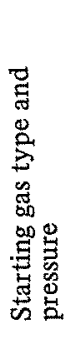 & 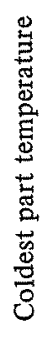 & 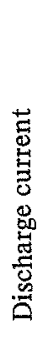 \\
\hline Starting time & 0 & 0 & 0 & & 0 & 0 & 0 & & & & & 0 & 0 & \\
\hline Starting voltage & 0 & $\mathrm{O}$ & & & & & 0 & 0 & $\mathrm{O}$ & & & 0 & & \\
\hline Restarting time & 0 & 0 & & & O & 0 & & 0 & O & & & O & 0 & \\
\hline Restarting voltage & & 0 & & & & & & 0 & $\mathrm{O}$ & & $\mathrm{O}$ & 0 & & \\
\hline Life (electrode, luminous flux holding) & $O$ & $\mathrm{O}$ & $\mathrm{O}$ & O & & O & $\mathrm{O}$ & 0 & & 0 & 0 & 0 & 0 & $\mathrm{O}$ \\
\hline Restriking voltage & & & 0 & 0 & & & 0 & 0 & & & $\mathrm{O}$ & 0 & & 0 \\
\hline Efficacy & 0 & $\mathrm{O}$ & O & & $\mathrm{O}$ & 0 & & & & & $\mathrm{O}$ & 0 & 0 & 0 \\
\hline $\begin{array}{l}\text { Luminous color } \\
\text { (color temperature, color rendering properties) }\end{array}$ & & & & & & & & & & 0 & $\mathrm{O}$ & & & $\mathrm{O}$ \\
\hline $\begin{array}{l}\text { Discharge stability } \\
\text { (acoustic resonance phenomenum) }\end{array}$ & (O) & (C) & (O) & & & & & & & & 0 & 0 & 0 & \\
\hline Electromagnetic wave emission & (C) & (C) & & & & & (0) & (C) & & & & & & \\
\hline Rectifying phenomenum & & & & & & & 0 & $\mathrm{O}$ & & & $\mathrm{O}$ & & & \\
\hline Dimming possibility & 0 & 0 & & & 0 & & 0 & & & & & & 0 & 0 \\
\hline
\end{tabular}

$\bigcirc$ Generally relating item

(O) Item to be considered in the electronic operating circuit

It is capable of raise the color temperature of the luminous tube by impressing a pulse current into the high pressure sodium lamp. That is because, by impressing the pulse current, the plasma temperature is raised, resulted in that the emission spectrum is extended to the short wavelength side by the $\mathrm{Na}$ emission from the higher excitation level and because $\mathrm{Hg}$ emission $(546 \mathrm{~nm})$ is added.

\subsubsection{Instant Restarting, Instant Stability}

It is desirable that the HID lamp must be restarted instant and stabilized instantly. Just after the putting-out of the lamp, the gas pressure in the inside of the luminous tube is high and the Pening Effect of $\mathrm{Hg}$ and rare gas has disappeared so that a high voltage is required for an instant restarting. Therefore, it will be effective for the lowering of restarting voltage to emit electron into the luminous tube by some means. It is a future task.

6.3 Future Trend of Electronic Operating Circuit and Discharge Lamp

(1) Dimming characteristic

Since at the high frequency operation the dimming can be performed by varying the frequency, the problem of restriking is not encountered so that a more stable dimming can be practiced than the prior system of using phase control. Although the problems relating to the discharge tube such as a moving striation at dimming and a discharge voltage rising are not the problems proper to the high frequency operation, they should be studied necessarily as a problem of lamp in order to correspond to the 
requirements of dimming.

(2) Tube diameter and fluorescent lamp characteristics

Since the electron temperature increase can be obtained by making the tube diameter thinner, it is advantageous to some extent from the point of efficacy to make the tube diameter thin. However, when a lamp with a thinner tube diameter is operated at a commercial power source frequency, the restriking voltage increases and the lamp power factor is lowered so that due to these problems the merit of thinner tube diameter cannot be attained necessarily in a sufficient degree. When operated at a high frequency, the above problems will not be encountered so that a thinning of tube diameter is advantageous. Furthermore, a thinning of tube diameter will provide together a merit of reduction of used material and compactness of light source, thereby the movement of thinning the tube diameter will be continued in future, supposedly. Therefore, it is desired for the study on the relation between the tube diameter and the fluorescent lamp characteristic including the problems relating to the starting voltage increase and the dimming characteristics.

(3) Operating frequency

As a frequency of high frequency operation, about $20 \mathrm{kHz}$ was used at first and recently about $50 \mathrm{kHz}$ is mainly used. It is estimated that this trend will be continued hereafter. In this case, it is the operating cirucit as well as the impeditive electromagnetic wave emission that present a great deal of problem. As a countermeasure against such problems, it is considered to use an electrodeless discharge by raising the frequency up to $\mathrm{MHz}$ region at a jump. From the electrodeless discharge in which electrodes are not required, a lot of characteristics can be expected. In addition, it is advantageous that, by using it, an acoustic resonance phenomenon can be removed from HID lamps. Therefore, a future study on the electrodeless discharge is expectable.

(4) High temperature restarting of HID lamp

It is expected that the electronic operating circuit for HID lamps will be mainly used for indoor service by utilizing its feature of lightweight. In this case, hot restarting is a strongly required feature. If hot restarting voltage below several $\mathrm{kV}$ would be attained, it will reach to the level of practical use. The progress of its study is expectable.

\section{Chapter 7 Postword}

References: 249 relating literatures officialized from Sept. 1986 to Dec. 1988 are listed up at the end of this Report.

\section{Postword}

It is estimated that a more variety of lighting systems will be designed and used in future towards the 21st century. In this progress the use of electronic device for the operating circuit and the discharge lamp adapted to the electronic operating circuit will take an important roll. It is our pleasure if this Committee Report will contribute at least to the development of this field. The Report is obtainable at a net charge from the Office of the Illuminating Engineering Institute of Japan.

Finally, I wish to thank the committee members and the relating persons for a great deal of efforts supplied during the committee-period. (Received July 6, 1989) 